\title{
HÁBITOS Y NECESIDADES DE CUIDADOS EN EL COLECTIVO DE EXTRANJEROS DE 80 Y MÁS AÑOS RESIDENTES EN LA COSTA MEDITERRÁNEA
}

D. RIVERA, A.REIG, M. CARRILLO, J.L. TALAVERA, A. CARUANA, C. FERRERA, A. MAJOS, R. CLIMENT, C. CLAVIJO, M. VERDÚ

Escuela Universitaria de Enfermería. Universidad de Alicante.

Hospital General de Alicante.

Facultad de Medicina de la Universidad de Alicante.

Ayuntamiento de Alicante.

Artículo publicado en la Revista GEROKOMOS, volumen IV, número 8, junio 1993. Primer Premio SEEG-B Braun Medical, S.A. V Congreso Nacional de Enfermería Geriátrica. Toledo. Abril 1993.

\section{RESUMEN}

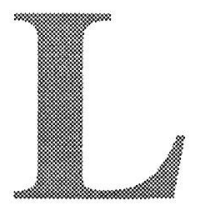

a costa mediterránea es el lugar del mundo donde habita un mayor número de ancianos en relación a la población total. La mayor parte, emigrantes venidos de otros países por razones climatológicas, económicas, etc. Con el paso del tiempo, el número de personas que superan los $80 \mathrm{y}$ más años es tan numeroso que puede rebasar a los recursos adecuados de atención. Nuestro estudio intenta detectar las necesidades socio-sanitarias de ese colectivo y presentar posibles vías de actuación.

Palabras clave: Anciano, extranjero, costa mediterránea, necesidades socio-sanitarias. 


\section{INTRODUCCIÓN}

\subsection{Justificación. ¿Por qué extranjeros? ¿Por qué 80 y más años?}

El litoral mediterráneo español es el lugar del mundo donde reside el mayor porcentaje de ancianos en relación a la población total. De los residentes extranjeros en España en las distintas regiones (sin contar las islas Canarias), el 37\% vive en la Comunidad Valenciana (el $32{ }^{\prime} 8 \%$ en Andalucía, el $20^{\prime} 1 \%$ en Baleares y el $10^{\prime} 1 \%$ en el resto del territorio español) y proceden casi en su totalidad de países europeos.

Por razones fundamentalmente climatológicas, geográficas, idiosincrásicas y económicas, las personas que se jubilan en Europa eligen con frecuencia afincarse en España, y esta tendencia puede mantenerse.

Con el paso del tiempo, estos emigrantes de la tercera edad se hacen mayores, lo que implica un incremento de la demanda de servicios, particularmente de salud y sociales. Concretamente, y según el Padrón Municipal de Habitantes de la Comunidad Valenciana de 1986, existían 84.745 extranjeros de 80 y más años residiendo en la Comunidad. Si multiplicamos esta cifra por 4 , ya que sabemos que por cada extranjero jubilado que se inscribe en el censo hay tres que no lo hacen, y si además añadimos todos aquellos que desde el año 1986 han cumplido 80 años, podemos hacernos una idea aproximada del colectivo de esta edad que está residiendo en algunos municipios de la Comunidad Valenciana, y suponer la cantidad y calidad de demanda real a los ayuntamientos respectivos.

Si se observan las estadísticas sobre residentes extranjeros mayores de 60 años y menores de 80 , se puede deducir que la situación presente es sólo el principio de algo que, siendo ya grave, va a aumentar constante y dramáticamente.

Nuestro país en general, la Comunidad Valenciana y sobre todo los municipios en donde la situación es más urgente no disponen actualmente de los recursos adecuados de atención. Ni siquiera se conoce con exactitud el número de personas extranjeras residentes. Se hace necesaria una oferta de recursos socio-sanitarios, de urbanismo y legislativos, principales problemas de organización con los que se enfrentan los jubilados extranjeros cuando han de buscar ayuda.

Nuestro trabajo se centra en uno de estos problemas, quizá el más importante para los ancianos: la salud. Salud entendida integralmente con la OMS como bienestar físico, psicológico y social. Las otras áreas, también importantes, sobrepasan nuestro principal foco de atención.

Cuando nuestros residentes extranjeros se hacen mayores y están solos, se encuentran con dos alternativas: en primer lugar, volver a su 
lugar de origen, lo que ya muchos países europeos tratan de evitar claramente con medidas legislativas. Estas naciones, debido a razones económicas prefieren mantener las pensiones de los jubilados que residen en España, a atenderles directamente en su última edad. La segunda alternativa para estos ancianos es quedarse aquí, quizá sin su pareja, quizá enfermos o necesitados de ayuda y sin saber dónde ni a quién acudir, entre otras razones porque ellos obvian su inscripción en el censo municipal y consulados.

Esta situación se ha de afrontar y ya, a fin de presentar posibles vías de actuación.

Nuestro trabajo intenta detectar las necesidades sociosanitarias de este colectivo cuya presencia en ciertos municipios de nuestra Comunidad es de una mayor entidad que en otras zonas del territorio nacional y europeo, y con características peculiares.

\subsection{Plan Integral de Atención Socio-sanitaria a la Tercera Edad}

Por otro lado, tanto la Generalitat como el gobierno de la nación están realizando un esfuerzo por adecuar los recursos existentes a las necesidades del colectivo mayor de 65 años. Nuestro trabajo se enmarca en varios aspectos importantes, en la línea del Plan Integral de Atención Socio-sanitaria a la Tercera Edad, elaborado por las Consejerías de Trabajo y Seguridad Social y Sanidad y Consumo.

\subsection{Municipalización y descentralización en cuanto a los criterios organizativos}

Dado que los problemas planteados por el colectivo extranjero de edad ocurren en localidades concretas, tratamos de colaborar en la iniciación de hecho, de una dinámica que priorice el municipio y su ayuntamiento como elemento organizador de los recursos según sus necesidades concretas y con vistas al futuro inmediato. Por esta razón, se ha proyectado el trabajo en Alfaz del Pi. Esta localidad reúne las características típicas de convivencia entre extranjeros de diversas nacionalidades y población autóctona.

\subsection{Jubilados europeos}

Estamos interesados particularmente en el colectivo de edad europeo, ya que su incidencia es determinante. La presencia de norteamericanos es muy escasa, y casi nula de emigrantes ancianos de los demás países del mundo. 


\subsection{Modelo de Atención Primaria}

Priorizamos las competencias de los Servicios Sociales Municipales y del Centro de Salud local en la atención primaria, inespecífica y generalizable a toda la población, particularmente en lo que se refiere a detección precoz y captación de personas y grupos de mayor riesgo en un intento de mantener exámenes periódicos de salud, atención a ancianos con perfil geriátrico (de 80 y más años y/o con graves problemas sociales), e intervención en el medio, organizando cuantas actividades socio-sanitarias sean precisas para favorecer la calidad de vida del anciano.

A partir de los datos que podamos aportar, se sugerirán alternativas de atención especializada, eficaces en esta localidad.

\subsection{Voluntariado}

Desde una perspectiva no ancianista, entendemos la ayuda de personas voluntarias, en nuestro caso de las diversas nacionalidades, como un elemento imprescindible. A estos grupos, es necesario organizarlos, coordinarlos y apoyarlos desde las instancias municipales de Servicios Sociales. La incidencia que pueden tener en la calidad de vida de la población anciana es inestimable.

Se intenta una dinámica de apoyo entre ancianos extranjeros, y constituir redes de apoyo socio-afectivo estables y eficaces.

\section{MATERIAL Y MÉTODOS}

\subsection{Procedimiento}

En primer lugar, se envió por correo una carta de presentación del proyecto, explicando los objetivos y pidiendo la colaboración de las personas extranjeras residentes en Alfaz del Pi. En ella, se adjuntaba el cuestionario de detección de necesidades socio-sanitarias. Tanto la carta explicativa como el cuestionario estaban redactados en el idioma del destinatario (en inglés, danés, alemán, holandés, noruego, sueco o francés). Además, se les anunciaba la visita de un compatriota voluntario una semana después, para ayudarle si lo necesitaba y recoger el cuestionario.

Al mismo tiempo, se envió una copia del cuestionario a los cónsules de las nacionalidades implicadas en dicho municipio, con el fin de que lo hiciesen llegar a sus ciudadanos no censados, pero inscritos en el consulado. 
Esta Primera Fase fue realizada durante los meses de enero, febrero y marzo de 1992. Los objetivos eran: 1) enviar un cuestionario sobre detección de necesidades, y 2) valorar la posible participación de personas de 80 y más años en un seguimiento de sus casos (programa de visitas).

Entre los 94 cuestionarios contestados, y a partir de la respuesta a si deseaban o no pertenecer al programa de visitas (que tiene como objetivos la evaluación precisa de necesidades socio-asistenciales y el seguimiento y ayuda a las personas extranjeras de 80 y más años), se formaron dos grupos:

a) Aquellos que no deseaban participar en el programa de visitas a domicilio (primera fase).

A estas personas se les envió por correo una carta en su idioma dando las gracias por contestar el primer cuestionario. De este grupo, seis personas cambiaron de opinión posteriormente y pidieron ser incluidos en el programa de visitas, quedando, finalmente, 42 personas.

b) Aquellos que deseaban ser incluidos en el programa (segunda fase). Para esta segunda fase, se organizó un equipo de «voluntarios» formado por personas extranjeras residentes en Alfaz del Pi. Los objetivos concretos al organizar la participación de voluntarios eran: a) facilitar la interrelación entre los voluntarios e integrarles en las actividades del proyecto en las diversas fases; b) conseguir la máxima participación e identificación con el proyecto, tanto de voluntarios como de ancianos de 80 y más años; c) trabajar conjuntamente en un clima de confianza para lograr una inserción en la realidad, evitando al máximo obstáculos de idiosincrasia, reservas y barreras idiomáticas.

\subsection{Instrumentos}

I. Actividades de la vida diaria.

II. Entrevista estructurada (de elaboración propia):

a) Hábitos alimenticios: 1) frecuencia con que se consumen productos lácteos, carnes, pescados, huevos, verduras y/o frutas, féculas, productos azucarados y productos grasos; 2) forma preferente de preparar los alimentos; 3) condimentos utilizados habitualmente; 4) cantidad aproximada de líquidos ingeridos durante el día; y 5) número de comidas realizadas diariamente.

b) Hábitos higiénicos: 1) forma y frecuencia de la higiene corporal; 2) cuidado de los pies.

c) Hábitos tóxicos: 1) consumo de tabaco; 2) alcohol; 3) bebidas estimulantes (café-té).

d) Ejercicio físico. 


\subsection{Sujetos}

La población de interés en el presente estudio son las personas extranjeras de 80 y más años residentes en el municipio de Alfaz del Pi y

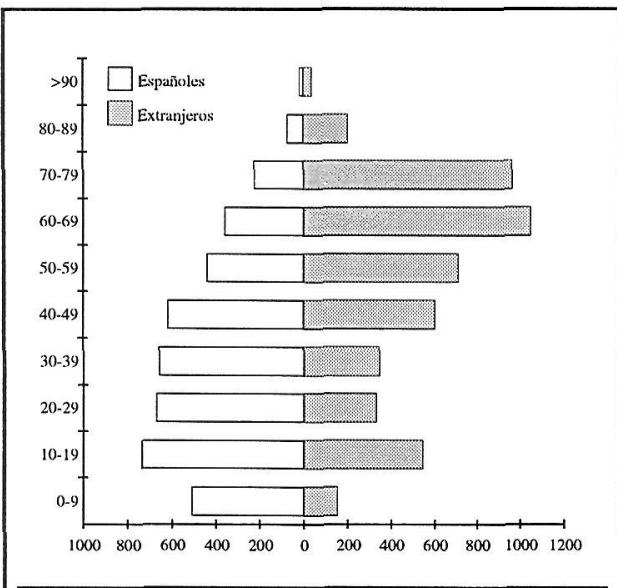

FIGURA 1: Distribución población censada Alfaz del Pi, diciembre, 1991 censadas en diciembre de 1991, así como algunas personas de estas características que sin estar censadas sí se encontraban inscritas en el consulado de su país.

En el censo de la población, en diciembre de 1991, se encontraron 4.699 ciudadanos extranjeros y 4.375 españoles (figura 1). Entre los extranjeros, 202 tienen 80 y más años, y 58 nacieron en 1912, se han incluido ya que a lo largo del estudio cumplirán los 80 .

Por tanto, nos hemos dirigido a 253 personas extranjeras de 80 y más años, de las que 139 son varones y 114 mujeres (figura 2).

Al cuestionario por correo, respondieron 94 personas. Han participado en la segunda fase (entrevistas domiciliarias) 34 personas.

\section{RESULTADOS}

\subsection{Actividades de la vida diaria}

Los resultados del índice de Katz, expuestos en la tabla 1, muestran el grado de independencia de las personas visitadas. Son totalmente independientes

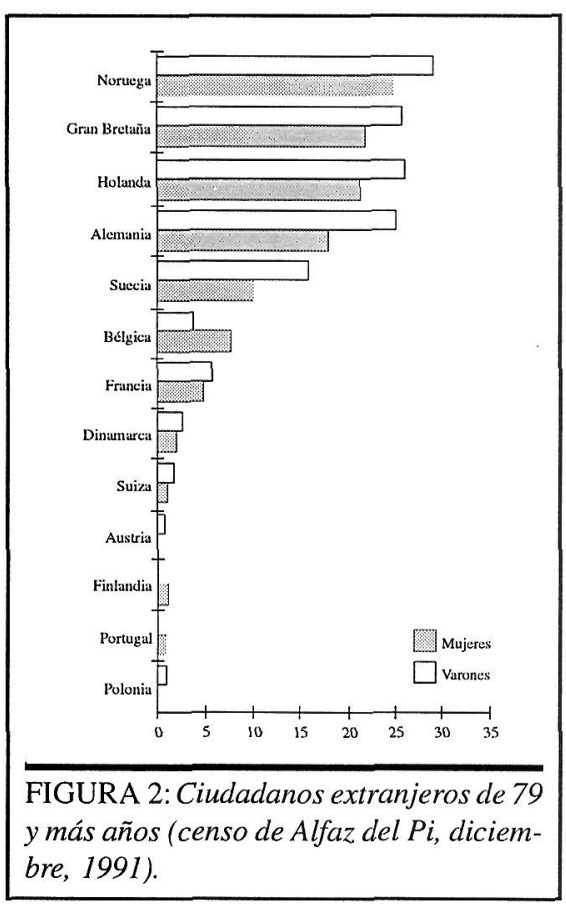




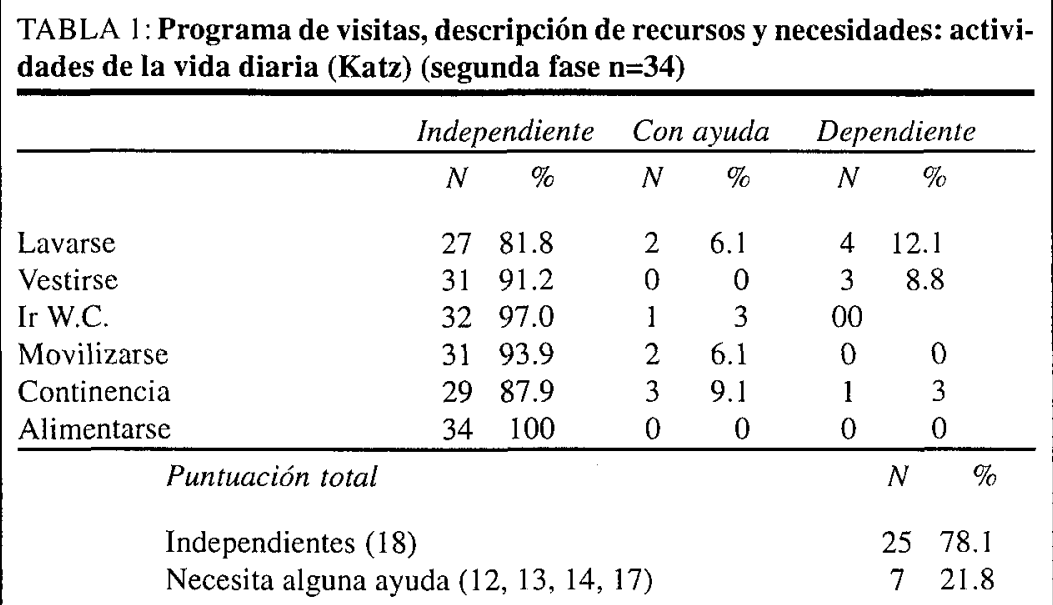

la mayoría $\left(78^{\prime} 1 \%\right)$, necesitando los demás alguna ayuda o presentando alguna dependencia particular. Concretamente, algunas personas necesitan ayuda para su incontinencia (9'1\%), movilizarse $\left(6^{\prime} 1 \%\right)$, lavarse $\left(6^{\prime} 1 \%\right)$ y/o ir al retrete $(3 \%)$. Otras son dependientes para lavarse $\left(12^{\prime} 1 \%\right)$, vestirse $(8 ' 8 \%)$ y/o para su continencia (3\%). Dada la edad de las personas visitadas, ese grado de independencia generalizado resulta muy positivo (tabla 1 ).

\subsection{Hábitos alimentarios}

En las tablas 2 y 3 se muestran los hábitos de consumo de líquidos y alimentos, así como la preparación de los mismos. El consumo de líquidos en general es de uno a dos litros $\left(96^{\prime} 9 \%\right)$. Se realizan habitualmente $\left(66^{\prime} 7 \%\right)$ tres comidas al día (desayuno, almuerzo y cena). Los alimentos se cocinan de una o dos maneras habitualmente $\left(69^{\prime} 7 \%\right)$.

TABLA 2: Programa de visitas, descripción de recursos y necesidades: hábitos alimentarios (segunda fase $n=34$ )

\begin{tabular}{lcccccrcr}
\hline & \multicolumn{2}{c}{ Diario } & \multicolumn{2}{c}{$2-3$ semana } & \multicolumn{2}{c}{1 semana } & \multicolumn{2}{c}{ Nunca } \\
\hline Frecuencia consumo & $N$ & $\%$ & $N$ & $\%$ & $N$ & $\%$ & $N$ & $\%$ \\
Leche & 29 & 87.9 & 0 & 0 & 2 & 6.1 & 2 & 6.1 \\
Carne & 19 & 57.6 & 12 & 36.4 & 2 & 6.1 & 0 & 0 \\
Pescado & 2 & 6.2 & 13 & 40.6 & 13 & 40.6 & 4 & 12.5 \\
Huevos & 3 & 9.7 & 13 & 41.9 & 9 & 29.0 & 6 & 19.4 \\
Verduras & 30 & 90.9 & 3 & 9.1 & 0 & 0 & 0 & 0 \\
Féculas & 25 & 75.8 & 3 & 9.1 & 4 & 12.1 & 1 & 3 \\
Azúcares & 20 & 64.5 & 1 & 3.2 & 4 & 12.9 & 6 & 19.4 \\
\hline
\end{tabular}




\begin{tabular}{|llccc|}
\hline $\begin{array}{l}\text { TABLA 3: Programa de visitas, descripción de recursos y necesidades: hábi- } \\
\text { tos de alimentación (segunda fse n=34) (continuación) }\end{array}$ & Número & N & \\
\hline & un modo & 11 & 33.3 \\
\hline Preparación preferente de los & dos modos & 12 & 36.4 \\
alimentos: fritos, cocidos, & tres modos & 6 & 18.2 \\
asados, guisados. & cuatro modos & 4 & 12.1 \\
& 0 & 2 & 6.2 \\
Número de condimentos que utiliza & 0 & 4 & 12.5 \\
habitualmente & 1 & 12 & 37.5 \\
& 2 & 10 & 31.2 \\
& 3 & 3 & 9.4 \\
& 4 & 1 & 3.1 \\
Cantidad de líquidos diarios & 5 & 11 & 33.3 \\
& 1 litro & 13 & 39.4 \\
& 1 l's litros & 8 & 24.2 \\
& 2 litros & 1 & 3.0 \\
Mínimo de comidas que realiza & $>2$ litros & 0 & 0 \\
al día: desayuno, almuerzo, & 1 & 06 & 18.2 \\
merienda y cena & 2 & 22 & 66.7 \\
& 3 & 5 & 15.2 \\
\hline
\end{tabular}

\begin{tabular}{|llccc|}
\hline $\begin{array}{l}\text { TABLA 4: Programa de visitas, descricpción de recursos y necesidades: hábi- } \\
\text { tos higiénicos (segunda fase } \mathbf{n = 3 4} \text { ) }\end{array}$ & & & \\
\hline & & $N$ & $\%$ \\
\hline En su higiene utiliza: & Baño & 7 & 21.9 \\
& Ducha & 22 & 68.7 \\
& Otros & 3 & 9.4 \\
Frecuencia de la higiene: & Mensual & 0 & 0 \\
& Quincenal & 3 & 9.4 \\
& Semanal & 9 & 28.1 \\
& Diaria & 20 & 62.5 \\
Cuidados de sus pies realizados por: & Ud. mismo & 12 & 37.5 \\
& Un familiar & 8 & 25.0 \\
& Un podólogo & 12 & 37.5 \\
& Semanal & 5 & 16.1 \\
& Quincenal & 7 & 22.6 \\
& Mensual & 19 & 61.3 \\
& Nunca & 0 & 0 \\
\hline
\end{tabular}

Los productos que se consumen más frecuentemente todos los días son, por orden de importancia, verduras (90'9\%), leche ( $\left.87^{\prime} 9 \%\right)$, féculas $\left(75^{\prime} 8 \%\right)$, seguidos de grasas $\left(69^{\prime} 7 \%\right)$, azúcares $\left(64^{\prime} 5 \%\right)$ y carne $\left(57^{\prime} 6 \%\right)$. 
Hay productos como huevos, azúcares, grasas y pescado que no se consumen nunca por un importante número de personas $\left(19^{\prime} 4 \%, 19^{\prime} 4 \%\right.$, $15 ' 2 \%$ y $12 ' 5 \%$, respectivamente). Los alimentos suelen acompañarse de uno o varios condimentos habitualmente $(812 \%)$ (tablas 2 y 3 ).

\subsection{Hábitos higiénicos}

En su higiene, las personas visitadas suelen utilizar la ducha $\left(68^{\prime} 7 \%\right)$ o, en menor medida, el baño (21'9\%), la mayoría diariamente (62'5\%) (tabla 4). El cuidado de sus pies lo realizan prácticamente todos ellos, bien acudiendo a un podólogo (37'5\%), bien haciéndolo ellos mismos $\left(37{ }^{\prime} 5 \%\right)$ o un familiar (25\%), preferentemente una vez al mes $(613 \%)$, a veces quincenalmente (22'6\%) o con frecuencia semanal (16'1\%) (tabla 4).

\subsection{Hábitos tóxicos}

El consumo habitual de tabaco es mínimo entre las personas visitadas $\left(15^{\prime} 2 \%\right)$, aunque la mayoría (54'5\%) son ex-fumadores (tabla 5). El consumo de alcohol está más extendido $\left(69^{\prime} 7 \%\right)$. Las personas que suelen tomar alcohol prefieren el vino (58'3\%), ya sea una o dos veces al día (42'3\%) u ocasionalmente (42'3\%) (tabla 5).

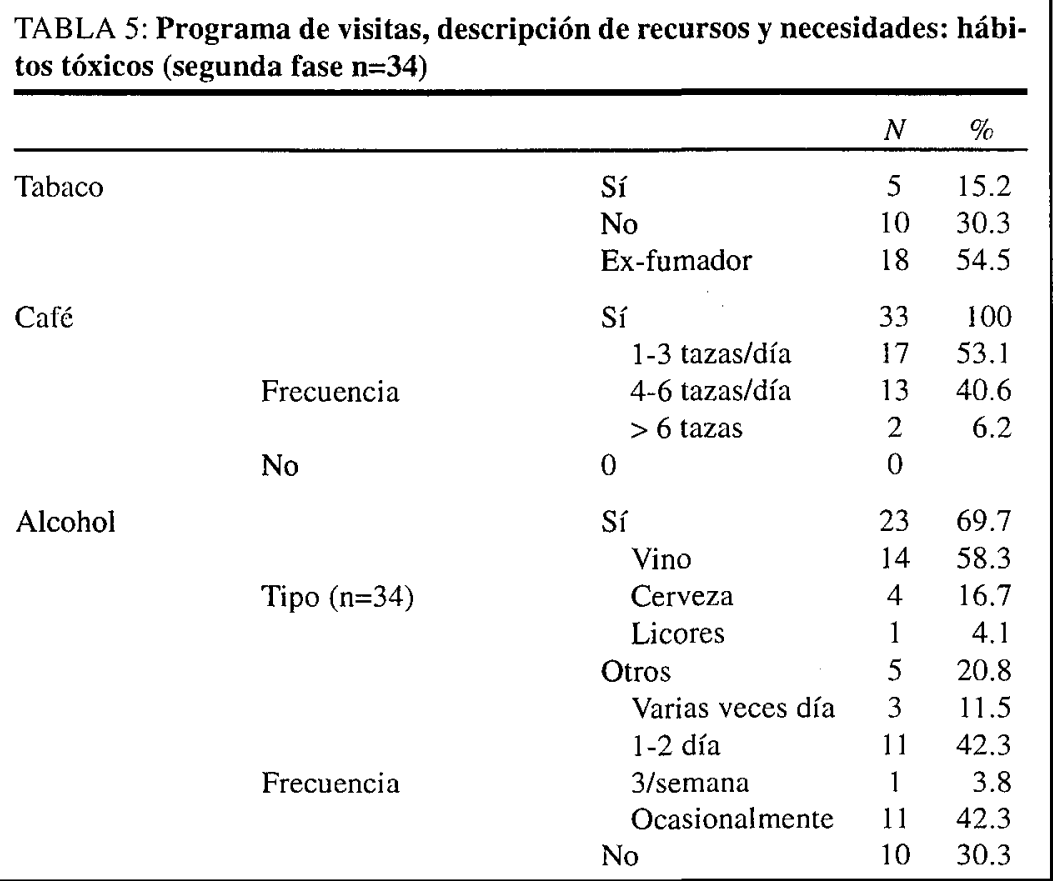




\subsection{Ejercicio físico}

Se observa que el $52 ' 94 \%$ de las personas visitadas (50\% varones, $50 \%$ mujeres) realizan algún tipo de ejercicio físico. Sólo algo más de la cuarta parte de los entrevistados (26,47\%) no lo realizan. Concretamente, pasean (32'3\%), hacen gimnasia (14'7\%), realizan trabajos de jardinería $(117 \%$ ) y otras actividades como baile, bicicleta o ejercicios de rehabilitación (11'6\%) (tabla 6).

\begin{tabular}{|c|c|c|c|c|c|c|}
\hline \multirow[b]{2}{*}{ Ejercicio físico } & \multicolumn{2}{|c|}{ Total } & \multicolumn{2}{|c|}{ Varones } & \multicolumn{2}{|c|}{ Mujeres } \\
\hline & $N$ & $\%$ & $N$ & $\%$ & $N$ & $\%$ \\
\hline Sí & 18 & 52.9 & 9 & 50 & 9 & 50 \\
\hline No & 9 & 26.4 & 4 & 44.4 & 5 & 55.5 \\
\hline No contesta & 7 & 20.5 & 3 & 42.8 & 4 & 57.1 \\
\hline \multicolumn{7}{|c|}{ Total } \\
\hline Ejercicios descritos & $N$ & $\%$ & & & & \\
\hline Pasear/andar & 11 & 32.3 & & & & \\
\hline Gimnasia & 5 & 14.7 & & & & \\
\hline Jardinería & 4 & 11.7 & & & & \\
\hline Baile & 2 & 5.8 & & & & \\
\hline Bicicleta & 1 & 2.9 & & & & \\
\hline Rehabilitación & 1 & 2.9 & & & & \\
\hline
\end{tabular}

\section{CONCLUSIONES}

De los datos expuestos en el capítulo de resultados, podemos concluir que la situación socio-sanitaria del colectivo de extranjeros de 80 y más años residente en Alfaz del Pi (Alicante) es bastante satisfactoria.

1) En efecto, la mayoría (78\%) son totalmente independientes.

2) Los hábitos alimentarios son bastante saludables, ya que su nutrición aparece en general equilibrada y completa, tanto en lo que se refiere a consumo de líquidos/día como de alimentos.

3) En cuanto a los hábitos higiénicos, casi todos utilizan la ducha o el baño habitualmente, y cuidan de un modo u otro sus pies.

4) El consumo de tabaco es inexistente (con bastantes ex-fumadores), aunque es muy frecuente tomar moderadamente vino en las comidas. El consumo de té está muy generalizado y es quizá excesivo.

5) Más de la mitad de este grupo acostumbra a realizar algún ejercicio físico. 
6) Aparece en los datos presentados un grupo de personas con problemas de dependencia física y necesidades de ayuda. Es importante el hecho de conocer quiénes son, dónde están ubicados y exactamente qué necesidades tienen. A través de esta investigación se han podido detectar y atender estos problemas individuales. Los Servicios Sociales municipales disponen ahora de una ficha individual de cada persona entrevistada con sus datos socio-demográficos y los factores de riesgo de salud y/o sociales.

7) La organización de la colaboración e implicación del grupo de voluntarios extranjeros, ha resultado un éxito, tanto para ellos mismos como para los ancianos con los que han contactado y para el futuro de tales contactos.

Independientemente de los datos expuestos y a partir de la experiencia de los miembros del equipo en la presente investigación, se deduce que los aspectos negativos encontrados, como problemas de salud, de funcionalidad, de aislamiento social, de incomunicación debido a los diversos idiomas, etc., implican una llamada de atención a la sociedad y a las instituciones, ya que el colectivo de extranjeros mayores de 60 años y menores de 80 es, en esta localidad (como en otras muchas de la costa de Alicante), diez veces más que los mayores de 80 . Estas personas irán cumpliendo años y presentando progresivamente más necesidades sociales y de salud.

La cuidadosa detección de necesidades de los ancianos, en particular de los muy mayores, es el primer paso para una precisa atención y rehabilitación de recursos. Nuestros datos pueden ser de utilidad a las instancias institucionales y políticas en sus negociaciones sobre recursos en la Comunidad Económica Europea.

Teniendo en cuenta los aspectos específicos comentados, sugerimos la necesidad de incrementar las redes asistenciales (incluyendo centros terminales, inexistentes por ahora en esta zona), coordinar y fomentar el voluntariado local y aumentar la capacitación gerontogeriátrica de los profesionales implicados en el cuidado de los ancianos.

\section{BIBLIOGRAFIA}

Conselleria de Treball i Seguretat Social. Plan Integral de Atención Sociosanitaria a la Tercera Edad. Dirección de Servicios Sociales. Area de Planificación de la Generalitat Valenciana. Valencia, 1991.

KATZ S., DOWNS T.D., CASH H.R., GROTZ R.C. Progress in development on the index of ADL. Gerontologist Spring, 1970; 1:20-30.

RIBERA D., MAJOS A., REIG A., TALAVERA J.L., FERRERA C., VERDÚ M., CLAVIJO C., CARUANA A. Identificación de necesidades socioasistenciales en el colectivo de extranjeros de la tercera edad residentes 

D. Ribera - A. Reig - M. Carrillo - J.L. Talavera - A. Caruana...

en la Comunidad Valenciana: un estudio en Alfaz del Pi con los extranjeros residentes de 80 y más años. Alicante, 1992. 